\title{
Secondary Postpartum Hemorrhage after Cesarean Delivery: A Case Report
}

\author{
Fatemeh Moshirinia $^{1}$, Mohaddeseh Motaharinejad ${ }^{2}$, Behjat Khorsandi*2
}

1. School of Nursing and Midwifery, Isfahan University of Medical Sciences, Isfahan, Iran

2. School of Nursing and Midwifery, Tehran University of Medical Sciences, Tehran, Iran

\begin{abstract}
Background: Delayed postpartum hemorrhage is a midwifery emergency that occurs following vaginal or cesarean delivery. It is one of the leading causes of postpartum hysterectomy and maternal death. The condition may be caused by dehiscence of uterus incision after cesarean section, which can lead to severe and fatal bleeding. We herein report a case of secondary postpartum hemorrhage after cesarean section.

Case description: A 34-year-old G3p3 woman with severe bleeding was admitted to the maternity hospital of Yazd (Iran) in preshock state. The patient had previously had three cesarean sections and underwent elective cesarean section at 38 weeks ten days age. The patient was immediately transferred to the operating room and underwent hysterectomy. Blood transfusion and serum therapy were performed. After the surgery, the patient was kept in the intensive care unit for four days and then was transferred to gynecology ward. Finally, she was discharged on the sixth day of hospitalization after partial recovery.

Conclusion: Secondary postpartum hemorrhage is a deadly complication of postpartum period. However, it is often diagnosed at critical stages due to its low incidence, which may endanger the mother's life if left unattended. Therefore, it is recommended to discharge all mothers after fully explaining the complications of genital infections, so that they may seek medical attention upon appearance of alarm symptoms. Gynecologists and health professionals should follow up this group of women more carefully to prevent irreversible consequences.
\end{abstract}

Keywords: Delayed hemorrhage, Delivery, Cesarean

$\begin{array}{lll}\text { Received: 2019/09/14 } & \text { Revised:2019/09/30 } & \text { Published:2019/10/03 }\end{array}$

*Correspondence: Behjat Khorsandi, School of Nursing and Midwifery, Tehran University of Medical

Sciences, Tehran, Iran

Tel: +98-9136089710

Email: bkhorsandi94@gmail.com 


\section{INTRODUCTION}

Postpartum hemorrhage is a midwifery emergency that occurs following vaginal or cesarean delivery (1). It is a major cause of postpartum hysterectomy and maternal death. Secondary postpartum hemorrhage is defined as any significant uterine bleeding occurring between 24 hours and 12 weeks postpartum but usually occurs 8 to 14 days after delivery (2). According to the World Health Organization, postpartum hemorrhage is responsible for about $25 \%$ of all maternal deaths (3). Postpartum hemorrhage could be atonic, traumatic or a combination of both. Investigations show that fetal macrosomia, induction or exacerbation of labor pain, magnesium sulfate use, chorioamnionitis and a history of postpartum hemorrhage can increase the risk of postpartum hemorrhage (4). Prenatal factors (history of bleeding in the current pregnancy, placenta previa, suspected or proven placental insufficiency, multiparity, excessive uterine dilatation due to polyhydramnios and macrosomia, preeclampsia, a history of retained placenta, maternal anemia, advanced maternal age), labor factors (macrosomia, episiotomy, labor induction, prolonged labor, emergency cesarean section), maternal coagulation disorders, obesity and body mass index of more than $40 \mathrm{~kg} / \mathrm{m}^{2}$ can also increase the risk of postpartum hemorrhage $(5,6)$. However, the most common cause of secondary postpartum hemorrhage is abnormal placental regression (7). Secondary hemorrhage after cesarean section is rare and occurs in about 1 per 365 cases (7). Secondary postpartum hemorrhage is characterized by severe bleeding, shock or anemia; therefore, proper diagnosis and management of bleeding is crucial (8). Depending on the severity and extent of bleeding, pharmacological, mechanical, non-surgical or surgical interventions may be applicable (9). Secondary postpartum hemorrhage and delayed bleeding can occur after dehiscence of uterus incision after cesarean section. In most cases, this condition results in severe bleeding in the second or third week after delivery, which often requires subtotal hysterectomy to control bleeding (8). We hereby report a case of delayed postpartum hemorrhage after cesarean delivery.

\section{CASE PRESENTATION}

A 34-year-old G3p3 woman with severe bleeding and in preshock state was admitted to the maternity hospital of Yazd (Iran) at midnight. The patient had previously had three cesarean sections and underwent elective cesarean section at 38 weeks ten days age. The patient had no pain or contraction in the hospitalization period for the cesarean section. There was no evidence of placental abnormality or adhesion in the Doppler ultrasound before delivery. After delivery and discharge, the patient had continuous bleeding for 10 days, but since the extent of bleeding resembled menstrual bleeding, the patient did not seek medical attention. She had no history of fever or abnormal, smelly discharge. The bleeding exacerbated with increased clots one hour before admission to the hospital. At time of admission, the patient had tachycardia (pulse rate: 130/min) and hypotension (7/4 $\mathrm{mmHg}$ ). Hemoglobin was $7.1 \mathrm{~g} / \mathrm{dl}$, hematocrit was $20.8 \%$ and platelet count was 272,000. Transfusion of $\mathrm{O}$ negative blood, Ringer's solution and oxytocin were administered concurrently. In addition, intramuscular vitamin $\mathrm{K}$ and intravenous tranexamic acid were administered. Due to severe bleeding and shock, the patient was immediately transferred to the operating room. After general anesthesia, laparotomy was performed by two gynecologists. About $500 \mathrm{ml}$ of diluted blood in the abdomen were suctioned. In the inspections, the uterine was soft, fragile and about 16 weeks old. There were numerous old clots that appeared to have accumulated in the uterus within the past few days. The previous cesarean section incision was open on the right. Finally, subtotal hysterectomy was performed due to severity of her condition, uncertainty about the exact location of bleeding and conditions of the uterus. During the operation, she received 8 units of packed cells, 8 units of fresh frozen plasma, 2 units of platelet, 3 liters of Ringer's 
solution, 3 liters of normal saline, $1 \mathrm{~g}$ of tranexamic acid, $200 \mathrm{mg}$ of hydrocortisone and a calcium ampoule. The patient was transferred to the intensive care unit (ICU) after recovery from the operating room. In the ICU, the patient received one unit of packed cells, serum therapy and antibiotic therapy. Complete blood count (CBC) was performed every six hours. Vital signs were normal. On the second day of hospitalization, an internist requested a peripheral blood smear due to platelet drop $(65,000)$. Schistocytes were observed in the peripheral blood smear. Fasting blood sugar, sodium, potassium, blood urea nitrogen, creatinine, SGOT, SGPT, lactate dehydrogenase and bilirubin were normal. After $\mathrm{CBC}$ was normalized (hemoglobin: $11.1 \mathrm{~g} / \mathrm{dl}$, hematocrit: $31.6 \%$, platelet: 105,000), she was transferred to the gynecology ward. The patient was treated with antibiotics and CBC was performed daily. Finally, she was discharged on the sixth day of hospitalization after partial recovery (hemoglobin: $11.8 \mathrm{~g} / \mathrm{dl}$, hematocrit: $33.6 \%$, platelet: 288,000$)$ and normal vital signs.

\section{DISCUSSION}

Postpartum hemorrhage is a midwifery emergency that may occur after both vaginal and cesarean delivery. Regardless of the definition of this condition, accurate diagnosis can be made by detection of a $10 \%$ postdelivery decline in hematocrit compared with pre-delivery (9). In our case, hematocrit was reduced to $20.7 \%$. Postpartum hemorrhage may occur within the first 24 hours of delivery or 24 hours to 12 weeks after delivery (10). Abnormal involution of the placental site is the most common cause of secondary postpartum hemorrhage (11), but rarely, cesarean wound dehiscence or nonunion of the incision site after cesarean section may also cause severe bleeding (7). Risk factors include diabetes, emergency surgery, infection and suturing technique. However, the exact cause of secondary postpartum hemorrhage sometimes cannot be identified (12). In these cases, ultrasound, blood culture and beta-HCG test (to rule out choriocarcinoma) are usually performed. It is recommended to directly perform exploratory laparotomy in cases with uterine scar dehiscence, shock and severe infection (7). In addition, pelvic arteriography is suggested for evaluation of vascular dysfunction (13). On exploratory laparotomy, the uterine incision may appear healthy or necrotic. In fact, hematoma, hypersensitivity to suture materials and posterior hematoma can lead to dehiscence of uterine wound (13). In a previous case report, the patient had no history of fever or abnormal discharge in the postpartum period. However, supra pubic and lower abdominal tenderness were noted. On the abdominal examination, scar was healthy and no abdominal mass was palpable. On pelvic examination, uterus was bulky and OS was closed. Ultrasound showed an endometrial thickness of $13 \mathrm{~mm}$. A complete dehiscence of the entire lower segment of uterine incision was noted. The uterus was enlarged to about 10-12 weeks pregnant size. During hysterectomy, swabs were taken from the margins of the uterine incision for culture, which later revealed infection with $E$. coli (14).

In our case, initial screening was not possible due to the emergency of the patient's condition and her preshock state. Therefore, the patient was immediately transferred to the operating room. Similar to the mentioned case, during the surgery, we noticed uterine scar dehiscence and several intrauterine blood clots. The uterus was soft, fragile and enlarged to about 16 weeks pregnant size. We were unable to identify the cause of delayed postpartum hemorrhage and the patient was subjected to subtotal hysterectomy. As mentioned above, risk factors for delayed postpartum hemorrhage are diabetes, emergency surgery, infection and suturing techniques. The patient had no history of emergency surgery or diabetes. The fragile and soft tissue at the incision opening-site proposes the possibility of infection.

\section{CONCLUSION}

Secondary postpartum hemorrhage is a deadly complication of postpartum period. However, it is often diagnosed at critical stages due to 
its low incidence, which endanger the mother's life if left unattended. Therefore, it is recommended to discharge all mothers after fully explaining the complications of genital infections, so that they may seek medical attention upon appearance of alarm symptoms. Gynecologists and health professionals should follow up this group of women more carefully to prevent irreversible consequences.

\section{DECLARATIONS \\ Funding \\ Not applicable}

\section{Ethics approvals and consent to participate}

Oral consent was obtained from the subject and she was assured that her personal data will remain confidential.

\section{Conflict of interest}

The authors declare that there is no conflict of interest regarding publication of this article.

\section{REFERENCES}

1. Sharma S, Gupta U, Kumar M, Tyagi S, Talwar N, Meena J, Singhal S, Pathak P. Pseudoaneurysm as a cause of secondary postpartum hemorrhage: a case report and literature review. Anatolian Journal of Obstetrics \& Gynecology. 2011; (4):14.

2. Dahlke JD, Mendez-Figueroa H, Maggio L, Hauspurg AK, Sperling JD, Chauhan SP, Rouse DJ. Prevention and management of postpartum hemorrhage: a comparison of 4 national guidelines. American journal of obstetrics and gynecology. 2015; 213(1):76-e1. [DOI:10.1016/ j.ajog.2015.02.023]

3. World Health Organization (2012) WHO Recommendations for the Prevention and Treatment of Postpartum Haemorrhage. World Health Organization, Geneva.

4. Royal College of Obstetricians and Gynaecologists (2011) Prevention and management of postpartum haemorrhage. Greentop Guideline, NO.52, p, 1-24

5. National Institute for health and care excellence (2014) Intrapartum care: care of healthy women and their babies during childbirth, NICE Clinical Guidelines [CG190].

6. Blomberg M. Maternal obesity and risk of postpartum hemorrhage. Obstetrics \&
Gynecology.

2011;118(3):561-8.

[DOI:10.1097/AOG.0b013e31822a6c59]

7. Wagner MS, Bédard MJ. Postpartum uterine wound dehiscence: a case report. J Obstet Gynaecol Can. 2006; 28(8):713-5. [DOI:10.1016/S1701-2163(16)32236-8]

8. Newsome J, Martin J G., Bercu Z., Shah J., Shekhani H , \& Peters G.. Postpartum hemorrhage. Techniques in vascular and interventional radiology. 2017; 20(4), 266-273.. [DOI:10.1053/j.tvir.2017.10.007]

9. Combs CA, Murphy EL, Laros JR. Factors associated with postpartum hemorrhage with vaginal birth. Obstetrics and gynecology. 1991;77(1):69-76.

10. Cunningham F, Leveno K, Bloom S, Spong CY, Dashe J. Williams obstetrics, 24e. Mcgrawhill; 2014. Page:800.

11. Tourné G, Collet F, Seffert P, Veyret C. Place of embolization of the uterine arteries in the management of post-partum haemorrhage: a study of 12 cases. European Journal of Obstetrics \& Gynecology and Reproductive Biology. 2003;110(1):29-34. [DOI:10.1016/S03012115(03)00091-5]

12. Vegas G, Illescas T, Muñoz M, Pérez-Piñar A. Selective pelvic arterial embolization in the management of obstetric hemorrhage. European Journal of Obstetrics \& Gynecology and Reproductive Biology. 2006;127(1):68-72. [DOI:10.1016/j.ejogrb.2005.09.008]

13. Nanjundan P, Rohilla M, Raveendran A, Jain $\mathrm{V}$, Khandelwal N. Pseudoaneurysm of uterine artery: a rare cause of secondary postpartum hemorrhage, managed with uterine artery embolisation. Journal of clinical imaging science. 2011;1(1):14.

14. Sengupta Dhar R, Misra R. Postpartum uterine wound dehiscence leading to secondary PPH: unusual sequelae. Case reports in obstetrics and gynecology. 2012;

[DOI:10.1155/2012/154685] 